\title{
Structural Insights into the Ligand Binding Domain of the Glucocorticoid Receptor: A Molecular Dynamics Study
}

\author{
N. R. Carina Alves, ${ }^{\S}$ Adali Pecci, ${ }^{\S, \dagger}$ and Lautaro D. Alvarez ${ }^{*}, \S, \ddagger$ \\ ${ }^{\S}$ Departamento de Química Biológica, Facultad de Ciencias Exactas y Naturales, Universidad de Buenos Aires, C1428EGA Buenos \\ Aires, Argentina \\ ${ }^{\dagger}$ CONICET-Universidad de Buenos Aires, IFIBYNE, C1428EGA Buenos Aires, Argentina \\ ${ }^{\ddagger}$ CONICET-Universidad de Buenos Aires, UMYMFOR, C1428EGA Buenos Aires, Argentina
}

Supporting Information

\begin{abstract}
The glucocorticoid receptor (GR) is a ligand-binding dependent transcription factor that ultimately regulates vital biological processes and inflammation response through specific gene expression control, thus representing a notable drug target to explore. Structurally, its ligand binding domain (LBD) harbors the region for the ligand-dependent transcriptional activation function 2 (AF-2), a majorly hydrophobic groove formed by residues from helices $\mathrm{H} 3, \mathrm{H} 4$, and $\mathrm{H} 12$, where the $\mathrm{H} 12$ position plays a critical role in AF-2 spatial conformation and GR function as a whole. However, the exact mechanisms underlying how regulatory ligands control the H12 structure and dynamics are yet to be elucidated. In this work, we have explored the correlation between ligand identity and GR LBD H12 behavior through different molecular dynamics (MD) simulations. After building diverse GR LBD systems in agonist and nonagonist states, we

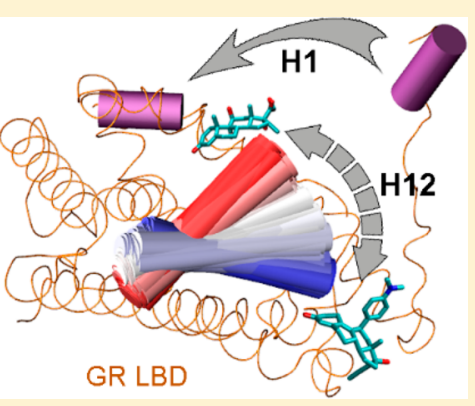
studied each system's response in the absence or the presence of an agonist ligand (dexamethasone) or an antagonist ligand (RU486) using classical MD simulations. We complemented them with steered MD for assessing the transition between those states and with the Umbrella Sampling method for free-energy evaluation. On the one hand, successfully obtaining fully folded nonagonist GR LBD states from the partially unfolded crystal GR LBD/RU486 underlines the role of the H1 in the GR LBD folding pathway. On the other hand, our results describe the H12 as a dynamic ensemble of conformations whose relative population is in the end determined by the interacting ligand: while dexamethasone privileges only a few poses (determined by a potential energy surface with a deep minimum), RU486 favors a wider $\mathrm{H} 12$ conformational amplitude, as indicated by a flatter potential landscape. By characterizing the H12 conformation in different conditions, we provide novel GR LBD models that represent potential targets for rational glucocorticoid drugs design, with the aim of accurately modulating GR activity.
\end{abstract}

\section{INTRODUCTION}

Growth, apoptosis, developmental behavior, and metabolism are among the biological features regulated by a group of steroid hormones named glucocorticoids (GCs). ${ }^{1,2}$ Given their powerful anti-inflammatory and immunosuppressive activities, they are administered as a treatment for asthma, dermatitis, rheumatoid arthritis, and other pathologies of autoimmune origin, while their proapoptotic nature extends GCs application to hematological neoplasms including various types of leukemias, lymphomas, and myelomas. ${ }^{3,4}$ This entitles GCs to be one of the most frequently prescribed medications worldwide. However, despite their evident benefits, GCs' clinical use is often limited by their severe long-term use side effects, e.g., osteoporosis, diabetes, hypertension, and obesity. Hence, developing new steroids that can dissociate therapeutic effects from adverse effects appears both promising and challenging, since it demands unraveling the molecular mechanisms underlying GCs physiological and pharmacological actions.

The glucocorticoid receptor (GR) is a transcription factor classified within the nuclear receptor (NR) superfamily ${ }^{6}$ and depicted as a modular protein with three prominent and conserved domains: an $\mathrm{N}$-terminal transactivation domain, a central DNA-binding domain (DBD), and a C-terminal ligandbinding domain (LBD). The $\mathrm{N}$-terminal domain contains the AF-1 transcriptional activation domain required for transcriptional enhancement and association with basal transcription factors. The DBD is composed of two zinc finger motifs which play a critical role in receptor homodimerization and DNA binding. The LBD consists of $11 \alpha$-helices $(\mathrm{H} 1 ; \mathrm{H} 3-\mathrm{H} 12)$ and four small $\beta$-strands folding into a globular structure, often described as a net, enclosing a central hydrophobic ligandbinding pocket $^{7}$ (LBP, Figure 1). Besides, LBD also harbors a second dimerization interface and the domain for the liganddependent transcriptional activation function 2 (AF-2). Particularly, the AF-2 domain is a majorly hydrophobic groove formed by residues from helices $\mathrm{H} 3, \mathrm{H} 4$, and $\mathrm{H} 12$, where the H12 position plays a critical role in the AF-2 spatial

Special Issue: Molecular Simulation in Latin America: Coming of Age

Received: September 12, 2019

Published: November 5, 2019 


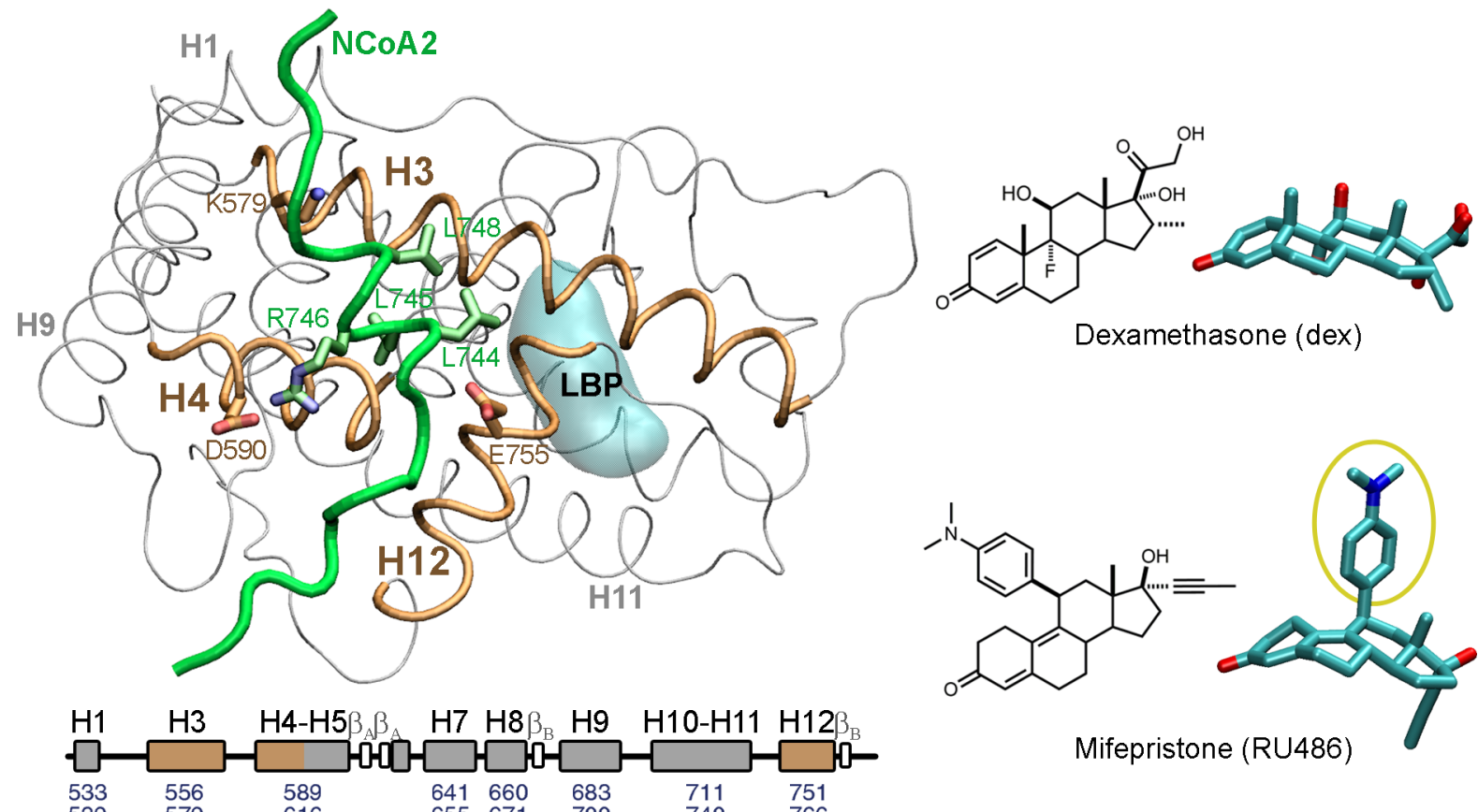

Figure 1. Left panel: three-dimensional structure of the GR LBD (pdb:1m2z, chain A) showing the H3, H4, and H12 helices forming the AF-2 domain which interacts with the NCoA2 peptide (in green). The position of the ligand binding pocket (LBP) is indicated as a cyan surface. A schematic of the secondary structure is shown below (numbers indicate first and final residues of each helix). Right panel: 2D and 3D structures of the glucocorticoid dexamethasone (dex) and the antiglucocorticoid mifepristone (RU486). The dimethylaniline moiety of RU486 is highlighted.

conformation and GR function. Indeed, the AF-2 domain interacts with specific nonpolar residues present in particular coregulators' amino acid motifs (LxxLL and I/LxxII for coactivators and corepressors families respectively, where $\mathrm{x}$ is any amino acid) arranged on one side of their amphipathic helix (Figure 1).

Hitherto, a central issue within the study of NRs is how regulatory ligands control the structure and dynamics of the H12. ${ }^{8,9}$ Initially, the analysis of the first crystallographic structures of isolated LBD led to propose the so-called induced-fit hypothesis-also known as the mouse-trap mechanism-to explain the molecular basis of action. This hypothesis postulates that, upon ligand binding, an inactive state of the receptor suffers a conformational change toward an agonist state. In the absence of a ligand (apo state), the H12 of several LBDs was described as a stable helix located away from the LBD body. ${ }^{10}$ However, further studies revealed that the $\mathrm{H} 12$ of apo-LBDs cannot be detached from the LBD surface and concluded that those observed positions were probably a consequence of artificial crystal packing interactions. ${ }^{11}$ Thus, in contrast to the induced-fit hypothesis, the conformational selection mechanism ${ }^{12}$ proposes that the receptor's apo state comprises an ensemble of conformations in which the $\mathrm{H} 12$ swings along different well-folded positions, and where ligandbinding drives the relative population of each subensemble. In this sense, by using fluorine-19 $\left({ }^{19} \mathrm{~F}\right)$ nuclear magnetic resonance (NMR), Chrisman et al. ${ }^{13}$ recently proved that the $\mathrm{H} 12$ of the nuclear receptor peroxisome proliferatoractivated receptor gamma (PPAR $\gamma)$ consists of a dynamic ensemble of conformations where the relative populations of subensembles correlate with the receptor's activity. Its AF-2 domain, including the $\mathrm{H} 12$, exchanges relatively fast between many conformations for the apo-receptor. In turn, agonist and inverse-agonist binding consolidates the complex's ensemble into structurally distinct active or inactive states, which favors coactivator or corepressor binding, respectively.

For the GR LBD, more than 40 crystallographic structures are archived in the Protein Data Bank (PDB, www.rcsb.org), mostly bound to ligands that stabilize the $\mathrm{H} 12$ in an agonist conformation. ${ }^{14}$ For nonagonist states, two structures of RU486 (Figure 1) complexes were resolved, ${ }^{15,16}$ providing information on the antagonistic mode of action of this antiglucocorticoid. In pdb:1nhz, the dimethylaniline group of RU486 appears to be preventing the $\mathrm{H} 12$ from adopting an agonistic conformation. However, this structure is incomplete and shows a fully deployed H12 bound to another GR LBD subunit. As described above, this is attributable to a crystallization technique's artifice. In turn, the case of pdb:3h52 tetrameric structure is more informative: the receptor is observed with the $\mathrm{H} 12$ in three different positions (all consistent with nonagonist states) where some allow the union of a peptide corresponding to a corepressor motif. Unfortunately, lacking residues at the $\mathrm{H} 11-\mathrm{H} 12$ region and an incomplete folding of the $\mathrm{H} 1$ and $\mathrm{H} 1-\mathrm{H} 3$ loop in the four monomers of pdb:3h52 prevent its direct use for further molecular modeling studies.

During the last decades, computational modeling techniques have enriched the study of proteins and constitute a profitable tool that complements and expands experimental data. ${ }^{17,18} \mathrm{~A}$ prominent example is the case of molecular dynamics (MD) simulation that can render protein's overall behavior in solution, thus allowing the study of protein inherent flexibility. Nevertheless, its robustness is limited in the presence of large energetic barriers between possible relevant conformations. To overcome this weakness, enhanced sampling methods have been developed, which expand and improve the understanding of the potential energy surface of a given system. ${ }^{19}$ In the case of NRs, MD simulation allowed for obtaining valuable 
Table 1. Summary of the Initial GR LBD Crystal Structures Used for cMD

\begin{tabular}{|c|c|c|c|c|}
\hline PDB ID/chain & $\mathrm{H} 1$ and $\mathrm{H} 1-\mathrm{H} 3$ loop & missing residues & peptide coregulator & receptor state \\
\hline $1 \mathrm{~m} 2 \mathrm{z} / \mathrm{A}$ & folded & & $\mathrm{NCoA} 2$ & GR LBD ${ }^{\mathrm{Ag}}$ \\
\hline 3h52/A & unfolded & 762-767 (H12 C-terminal loop) & NCoR1 & GR LBD ${ }^{\text {Ant1 }}$ \\
\hline $3 \mathrm{~h} 52 / \mathrm{B}$ & unfolded & & NCoR1 & GR LBD ${ }^{\text {Ant2 }}$ \\
\hline $3 \mathrm{~h} 52 / \mathrm{C}$ & unfolded & $738-754$ (part of H11; H11-H12 loop; part of H12) & & GR LBD ${ }^{\text {Ant3 }}$ \\
\hline $3 \mathrm{~h} 52 / \mathrm{D}$ & unfolded & $742-750(\mathrm{H} 11-\mathrm{H} 12$ loop $)$ and $762-765(\mathrm{H} 12)$ & & GR LBD ${ }^{\text {Antl }}$ \\
\hline
\end{tabular}
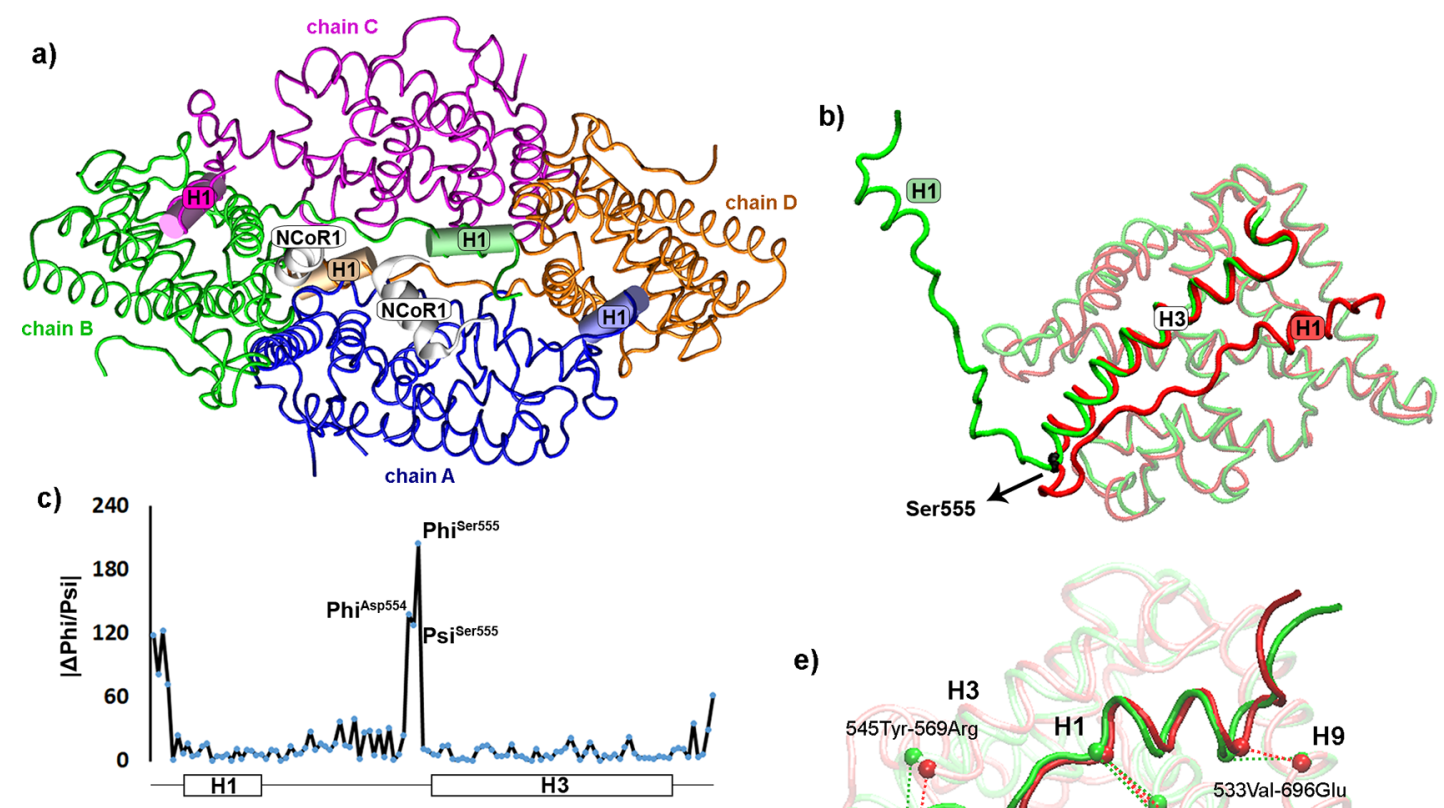

d)
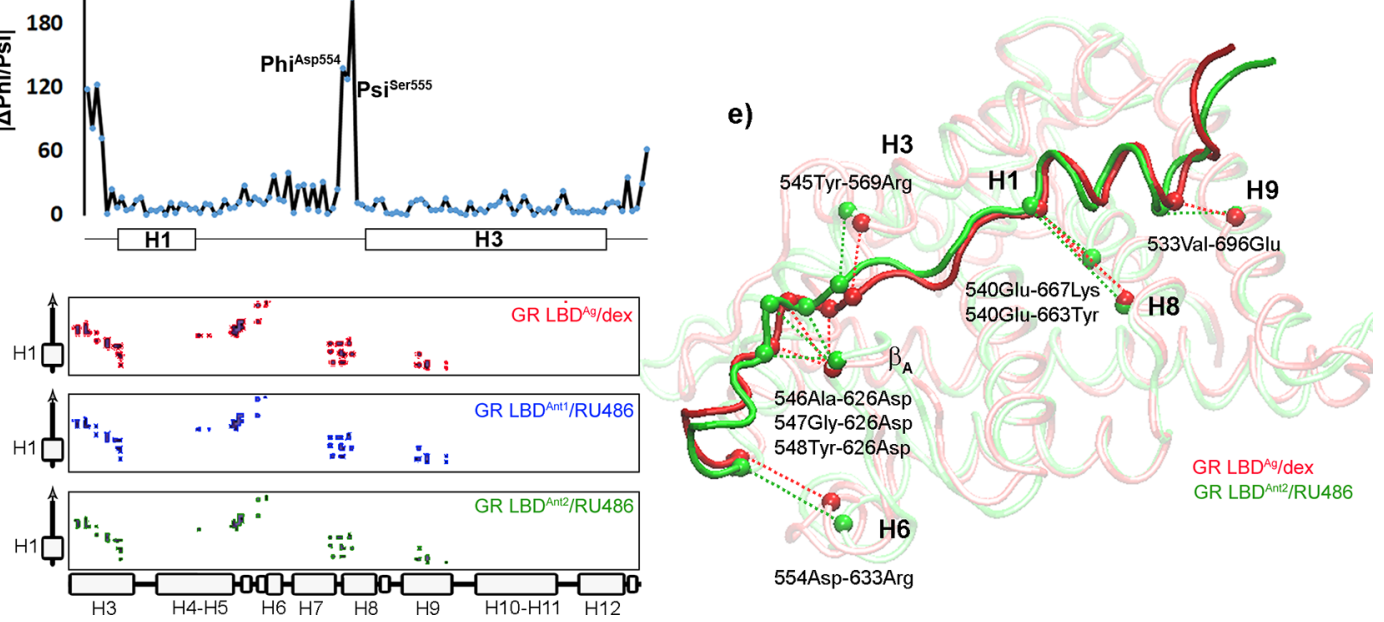

Figure 2. a) Overall view of the GR LBD/RU486 tetramer ( $\mathrm{pdb}: 3 \mathrm{~h} 52$ ): chain A in blue, B in green, C in magenta, and D in orange. H1 helices are schematized as cylinders, and NCoR1 peptides are shown in white. b) Superposition of chain A of pdb:1m2z (red) and chain B of pdb:3h52 (green). c) Absolute value of the difference between the Phi and Psi angles of the H1 and H1-H3 loop segment in pdb:1m2z (chain A) and pdb:3h52 (chain B). d) Contact map between residues from the $\mathrm{H} 1$ and $\mathrm{H} 1-\mathrm{H} 3$ loop and the remainder residues of the receptor for the equilibrated complexes. e) Main contacts between the $\mathrm{H} 1$ and $\mathrm{H} 1-\mathrm{H} 3$ loop and the remainder of the receptor. $\mathrm{C} \alpha$ of the involved residues are shown as spheres.

information regarding the ligand binding mode, the binding and unbinding pathways, and their molecular basis of action. ${ }^{20}$ Moreover, research on the dynamic association between the $\mathrm{H} 12$ position and the action of ligands bound to the LBP was recently conducted for other steroid receptors: progesterone, ${ }^{21}$ androgen, ${ }^{22,23}$ and estrogen ${ }^{24}$ receptors. However, to our knowledge, no studies were dedicated to thoroughly analyzing the conformational equilibria of the H12 in the GR LBD.

The above concerns encouraged us to explore through different MD simulation methods the correlation between ligand identity and the GR LBD $\mathrm{H} 12$ behavior. With this purpose, we constructed and studied several systems. The absence or the presence of an agonist ligand (dexamethasone) or an antagonist ligand (RU486) was combined with GR LBD both in agonist and nonagonist states. We envisaged that dissimilar starting conformations for the classical MD (cMD) analysis could offer a wider panorama on the H12 dynamics, while including a combination of steered MD (SMD) and the Umbrella Sampling (US) method would provide additional insights into the molecular determinants involved in the transition between those states.

\section{RESULTS}

2.1. Construction of GR LBDs in Nonagonist States. The first aim of this work focused on the in silico construction of GR LBDs in nonagonist states, to obtain viable structures for MD simulation studies. As previously mentioned, monomers from pdb:3h52 have several remarkable $\mathrm{H} 12$ conformations, but inconveniently, their N-terminal end ( $\mathrm{H} 1$ and $\mathrm{H} 1-\mathrm{H} 3$ loop) is unfolded from the LBD body and packaged in other monomers of the unit cell (Table 1 and Figure 2a). Although at first sight this phenomenon was associated with a crystallization artifice, ${ }^{16}$ a recent study by Suren et al. ${ }^{25}$ using single-molecule force spectroscopy showed 
that the $\mathrm{H} 1$ folding, in fact, comprises the last step in the overall folding process and occurs only upon ligand binding. Thus, monomers of pdb:3h52 structure could presumably correspond to the open state described by these authors, constituting an intermediate state receptive to ligand binding. On this basis, we considered it feasible to obtain completely folded states of nonagonist systems starting from $\mathrm{pdb}: 3 \mathrm{~h} 52$ structures.

The structural alignment between pdb: $1 \mathrm{~m} 2 \mathrm{z}$ and $\mathrm{pdb}: 3 \mathrm{~h} 52$ reveals a large congruence among most GR LBD helices (Figure 2b). Indeed, their conformation concords from residue $555(\mathrm{H} 3)$ onward, except only for the $\mathrm{H} 11-\mathrm{H} 12$ region where the helices exhibit slightly different positions (see below). After comparing $\mathrm{H} 1$ and $\mathrm{H} 1-\mathrm{H} 3$ loop backbone dihedral angles Phi and Psi between the fully folded and the partially unfolded structures, it was noticed that only three angles at the end of the H1-H3 loop (PhiAsp554, PsiAsp554, and PhiSer555) diverged (Figure 2c). Apart from the pointed differences, no major changes were found along the rest of the loop or helices $\mathrm{H} 1$ and H3. Thus, after having established the structural similarity between both complexes, we obtained fully folded nonagonist state systems by replacing the coordinates of residues 528 to 554 in pdb:3h52 chains with those from the corresponding residues of chain $A$ in the pdb: $1 \mathrm{~m} 2 \mathrm{z}$ structure.

Each monomer from pdb:3h52 was then analyzed separately. In chain $\mathrm{A}$ (termed here as GR LBD ${ }^{\mathrm{Ant} 1}$ ), there were 6 missing residues following the H12 C-terminal loop (Table 1), which we added using the Modeller software. In chain B (termed here as GR LBD ${ }^{\text {Ant2 }}$ ), the GR LBD was fully resolved. The structural alignment between both nonagonist GR LBD states here constructed (GR LBD ${ }^{\text {Ant1 }}$ and GR $\mathrm{LBD}^{\text {Ant2 }}$ ) and the agonist system $\left(\mathrm{GR} L B D^{\mathrm{Ag}}\right.$ ) displays differences in the $\mathrm{H} 12$ position (Figure 3a). On the other hand, chain $\mathrm{C}$ (termed here as GR $\mathrm{LBD}^{\mathrm{Ant} 3}$ ) revealed a noteworthy $\mathrm{H} 12$ position displaced to the opposite side of the RU486 $11 \beta$-substituent compared with the GR LBD ${ }^{\text {Ant1 }}$ and GR LBD ${ }^{\text {Ant2 }}$, partially occupying the AF-2 groove (Figure $3 \mathrm{~b}$ ). Unfortunately, this helix was poorly resolved, with as much as 17 residues missing along the H11 C-terminal end, the H11-H12 loop, and part of H12. We fruitlessly attempted to model this large portion of the receptor with Modeller, but no convincing structures were achieved. Lastly, chain D, with 13 unresolved residues along the H11H12 loop and H12, showed a H12 configuration equivalent to the observed in chain A (GR LBD $\left.{ }^{A n t 1}\right)$. Preliminary MD simulations from these two models showed a high structural instability, thus, in view of the above, neither chain $\mathrm{C}$ nor $\mathrm{D}$ was further considered in this study.

2.2. Initial Evaluation of Nonagonist GR LBDs. GR $\mathrm{LBD}^{\text {Ant1 }} / \mathrm{RU} 486$ and GR $\mathrm{LBD}^{\text {Ant2 }} / \mathrm{RU} 486$ complexes were energetically minimized and equilibrated through classical MD simulations. The Ramachandran plots of the resulting structures show that nearly all residues are present in the favored regions, few are present in the permitted ones, and only one is present in a forbidden region (Figure S1). Besides, the secondary structure of the modified section remaining comparable to that of GR $\mathrm{LBD}^{\mathrm{Ag}}$, the $\mathrm{H} 1$ rearrangement was notably stabilized in the designated position, and the $\mathrm{H} 1-\mathrm{H} 3$ loop did not suffer substantial structural deformations. Moreover, the analysis of the contacts between the N-terminal end and the remainder of the receptor revealed a high similarity between the original GR $\mathrm{LBD}^{\mathrm{Ag}}$ and the constructed GR LBD ${ }^{\text {Ant1 }}$ and GR LBD ${ }^{\text {Ant2 }}$ systems (Figure $2 \mathrm{~d}$ and e).

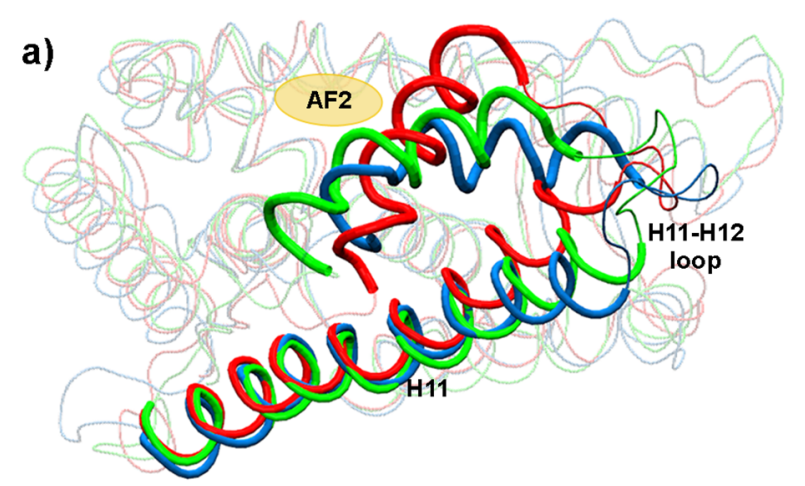

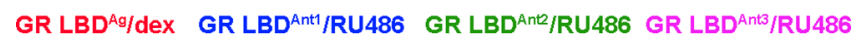

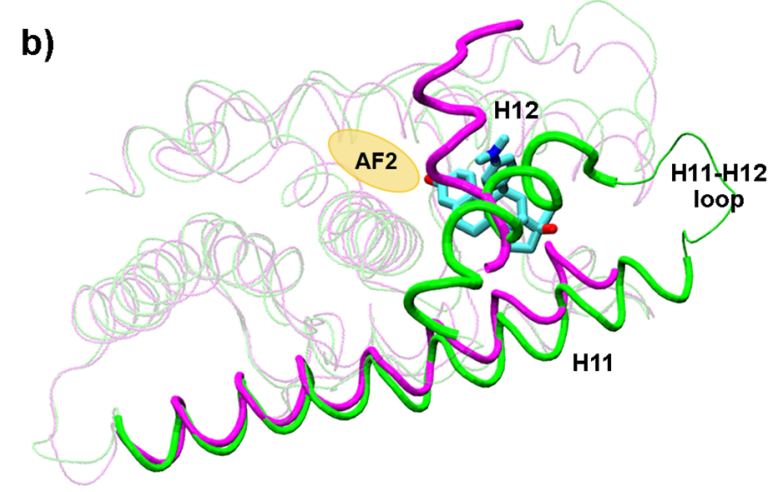

Figure 3. a) Superposition of the initial structures of GR LBD ${ }^{\mathrm{Ag}}$, GR LBD $^{\text {Ant1 }}$, and GR LBD ${ }^{\text {Ant2 }}$ evidencing H12 different positions. b) Superposition of chain B $\left(G R L^{2} D^{A n t 2}\right)$ and chain C $\left(G R L^{2} D^{A n t 3}\right)$ of pdb:3h52. RU486 is colored in cyan. In both figures, the H11, the $\mathrm{H} 12$, and the H11-H12 loop are highlighted, and the position of the AF2 groove is indicated with a yellow ellipse.

Finally, a proper H12 helical conformation was also conserved (see below).

2.3. Classical Molecular Dynamics Simulation of GR LBD Systems. Once the quality of the initial GR LBD models was evaluated, we simulated the systems GR $\mathrm{LBD}^{\mathrm{Ag}}$, GR $\mathrm{LBD}^{\text {Ant1 }}$, and GR LBD ${ }^{\text {Ant2 }}$ through $500 \mathrm{~ns}$ cMD to study the dynamic behavior under different conditions (Table 2), i.e., in the presence of the corresponding ligand (dex for GR LBD ${ }^{\mathrm{Ag}}$; RU486 for GR LBD ${ }^{\text {Ant1 }}$ and GR LBD ${ }^{\text {Ant2 }}$ ), in the absence of a ligand (apo systems), and in the presence of the opposite ligand (RU486 for GR LBD ${ }^{\mathrm{Ag}}$; dex for GR LBD ${ }^{\mathrm{Ant} 1}$ and GR $\mathrm{LBD}^{\text {Ant2 }}$.

2.3.1. Stability and Fluctuation Pattern of CMD Trajectories. The stability of $\mathrm{cMD}$ trajectories was then assessed by monitoring backbone atoms' RMSD relative to the initial structures. It was noticed that while during the first 200 ns the protein structure evidenced fluctuations, all GR $\mathrm{LBD}^{\mathrm{Ag}}$ and GR LBD ${ }^{\text {Ant2 }}$ systems were stabilized thereafter in constant values, which were less than $2.2 \AA$ (Figure 4a). In turn, the GR $\mathrm{LBD}^{\text {Ant1 }}$ systems suffered significant deviations all along the simulation, and only for the GR LBD ${ }^{\text {Ant1 }} /$ RU486 the trajectory seemed to converge. On the other hand, the RMSD of the H12 $\mathrm{C} \alpha$ atoms (Figure 4b) showed that from $200 \mathrm{~ns}$ onward the H12 position was stabilized in all systems, except for GR $\mathrm{LBD}^{\text {Ant1 }}$ /apo. Specifically, in systems GR $\mathrm{LBD}^{\mathrm{Ag}}$ and GR LBD $^{\text {Ant1 }}$, the stabilized $\mathrm{H} 12$ was located close to the departing position when their original ligands were involved (dex and RU486, respectively). For GR $\mathrm{LBD}^{\mathrm{Ag}}$, the introduction of RU486 slightly deviated the H12, while the removal of the 
Table 2. Simulated Systems and Average Distances between "Charge Clamp” Residues

\begin{tabular}{|c|c|c|c|c|c|}
\hline \multirow[b]{2}{*}{ system } & \multirow[b]{2}{*}{ receptor } & \multirow[b]{2}{*}{ ligand } & \multicolumn{3}{|c|}{ distance $(\AA)^{a}$} \\
\hline & & & Glu755-Lys579 & Glu755-Asp590 & Asp590-Lys579 \\
\hline 1 & GR LBD ${ }^{A g}$ & Dex & 18.4 & 15.0 & 11.8 \\
\hline 2 & GR $\mathrm{LBD}^{\mathrm{Ag}}$ & none & 18.3 & 15.0 & 11.7 \\
\hline 3 & GR LBD ${ }^{\mathrm{Ag}}$ & RU486 & 17.8 & 12.9 & 11.9 \\
\hline 4 & GR LBD ${ }^{\text {Antl }}$ & RU486 & 22.2 & 21.6 & 12.0 \\
\hline 5 & GR LBD ${ }^{\text {Antl }}$ & none & 21.5 & 20.4 & 11.7 \\
\hline 6 & GR LBD ${ }^{\text {Antl }}$ & Dex & 20.5 & 20.2 & 11.9 \\
\hline 7 & GR LBD ${ }^{\text {Ant2 }}$ & RU486 & 20.1 & 16.6 & 11.6 \\
\hline 8 & GR LBD ${ }^{\text {Ant2 }}$ & none & 18.5 & 15.5 & 11.4 \\
\hline 9 & GR LBD ${ }^{\text {Ant2 }}$ & Dex & 18.4 & 15.2 & 11.4 \\
\hline
\end{tabular}

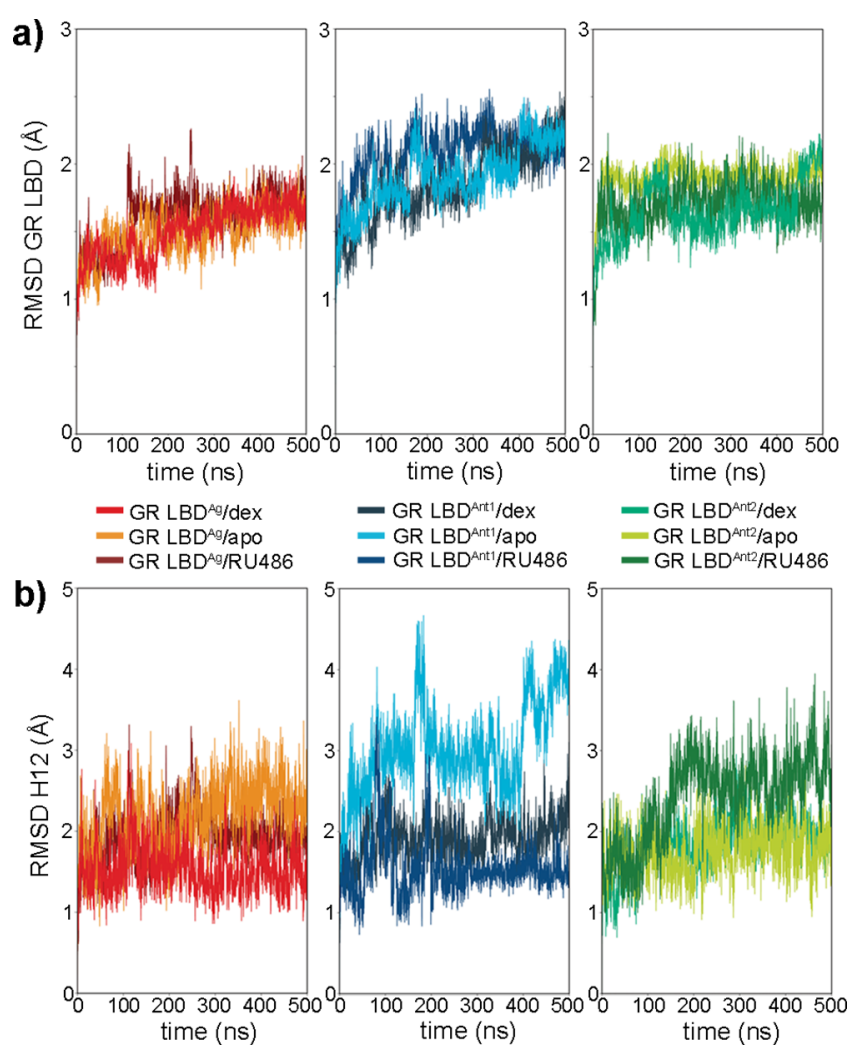

Figure 4. Root mean square deviation (RMSD) of the 9 simulated systems from the initial structures over all backbone atoms (a) or over H12 backbone atoms (b).

ligand considerably deflected it. Likewise, in GR LBD ${ }^{\text {Ant1 }}$, the absence of a ligand provoked a remarkable distortion of the $\mathrm{H} 12$ position.

The RMSF calculated over the last $300 \mathrm{~ns}$ from the simulation (Figure S2) revealed, besides loops' inherent mobility, some regions of secondary structure with meaningful differences among systems. Regarding the C-terminal end, the $\mathrm{GR} \mathrm{LBD}^{\mathrm{Ag}} /$ dex complex showed the least mobility, and the ligand's substitution by RU486 clearly increased the fluctuation of the H11-H12 loop and the H12 first residues. A different RMSF profile was observed for the GR $\mathrm{LBD}^{\text {Ant1 }}$ systems, with a large mobility in the C-terminal end of the H12. Among them, the GR $\mathrm{LBD}^{\text {Ant1 }} / \mathrm{RU} 486$ appeared the most rigid; GR $\mathrm{LBD}^{\mathrm{Ant1}}$ /apo presented an increment in $\mathrm{H} 12$ mobility, and GR $\mathrm{LBD}^{\mathrm{Ant} 1} / \mathrm{dex}$ had the $\mathrm{H} 11-\mathrm{H} 12$ loop fluctuation augmented. In turn, the RMSF pattern of GR LBD ${ }^{\text {Ant2 }}$ complexes resembled those of the GR $\mathrm{LBD}^{\mathrm{Ag}}$ systems, with some increased fluctuation for the GR $\mathrm{LBD}^{\mathrm{Ant} / 2} / \mathrm{dex} \mathrm{H} 12$ residues.

2.3.2. Evolution of the H12 Conformation in the $C M D$ Trajectories. A detailed RSMF global analysis highlighted the great stability of helices $\mathrm{H} 3$ and $\mathrm{H} 10-\mathrm{H} 11$ along the cMD, particularly of the $\mathrm{H} 3 \mathrm{C}$-terminal end (residues 568 to 579; hereinafter, portion " $\mathrm{B}$ " or $\mathrm{H} 3 \mathrm{~B}$ ) and the $\mathrm{H} 10-\mathrm{H} 11 \mathrm{~N}$ terminal residues 711 to 721 (hereinafter, H10). This peculiarity allowed us to select these virtually invariant segments as a spatial reference to analyze the mobility of the H12 over time. Three vectors of equal direction than segments $\mathrm{H} 3 \mathrm{~B}, \mathrm{H} 10$, and helix $\mathrm{H} 12$ were defined, and the resulting angles were calculated along the complete $\mathrm{cMD}$. In accordance with the cited RMSD analysis, the temporal evolution of these angles evidenced that all systems converged after the $200 \mathrm{~ns}$ (Figure S3) with the exception of GR $\mathrm{LBD}^{\text {Ant1/ }}$ apo that presented great instability at the $\mathrm{H} 3 \mathrm{~B}-\mathrm{H} 12$ angle.

For visualization purposes, the last 300 ns of each cMD trajectory were then examined using box plots (Figure 5). As expected, the $\mathrm{H} 3 \mathrm{~B}-\mathrm{H} 10$ angle was mainly conserved among the 9 systems, particularly within GR LBD ${ }^{\mathrm{Ag}}$ and GR LBD ${ }^{\mathrm{Ant} 2}$ complexes. Conversely, angles $\mathrm{H} 3 \mathrm{~B}-\mathrm{H} 12$ and $\mathrm{H} 10-\mathrm{H} 12$ displayed a more condition-dependent behavior. This was particularly evident for the GR LBD ${ }^{\text {Ant1 }}$ systems, where large changes were observed in both angles when RU486 was removed from the LBP or replaced by dex. Although less pronounced, the GR $\mathrm{LBD}^{\mathrm{Ant2}}$ systems also showed certain differences in the evolution of those angles. Lastly, for GR $\mathrm{LBD}^{\mathrm{Ag}}$ systems, both angles suffer only narrow deviations, and solely the imposed presence of RU486 markedly disturbed angle $\mathrm{H} 3 \mathrm{~B}-\mathrm{H} 12$.

For further characterization of the stabilized $\mathrm{H} 12$ conformation, we calculated the spatial distribution of the angles $\mathrm{H} 3 \mathrm{~B}-\mathrm{H} 12$ and $\mathrm{H} 10-\mathrm{H} 12$ over the last $300 \mathrm{~ns}$ of the simulation and plotted them as $2 \mathrm{D}$ histograms (Figure 6a). There, different $\mathrm{H} 12$ states achieved by each system are clearly displayed. In the case of the $\mathrm{GR} \mathrm{LBD}^{\mathrm{Ag}}$, there was a tight distribution of $\mathrm{H} 3 \mathrm{~B}-\mathrm{H} 12$ and $\mathrm{H} 10-\mathrm{H} 12$ angles, around $75^{\circ}$ and $98^{\circ}$, respectively, corresponding to the $\mathrm{GR} \mathrm{LBD}^{\mathrm{Ag}} / \mathrm{dex}$ state. H12 disposition was not perceptibly altered after dex removal, but its substitution by RU486 slightly withdrew the receptor from the agonist state. However, the H12 did not turn toward any of the positions observed for complexes GR $\mathrm{LBD}^{\text {Ant1 }}$ or GR $\mathrm{LBD}^{\text {Ant2 }}$ but in the opposite direction, interestingly resembling that of the GR LBD ${ }^{\text {Ant3 }}$ state (cf. Figure $3 \mathrm{~b}$ ). This led us to evaluate $\mathrm{GR} \mathrm{LBD}^{\mathrm{Ag}} / \mathrm{RU} 486$ possible additional conformational changes. We extended its trajectory 

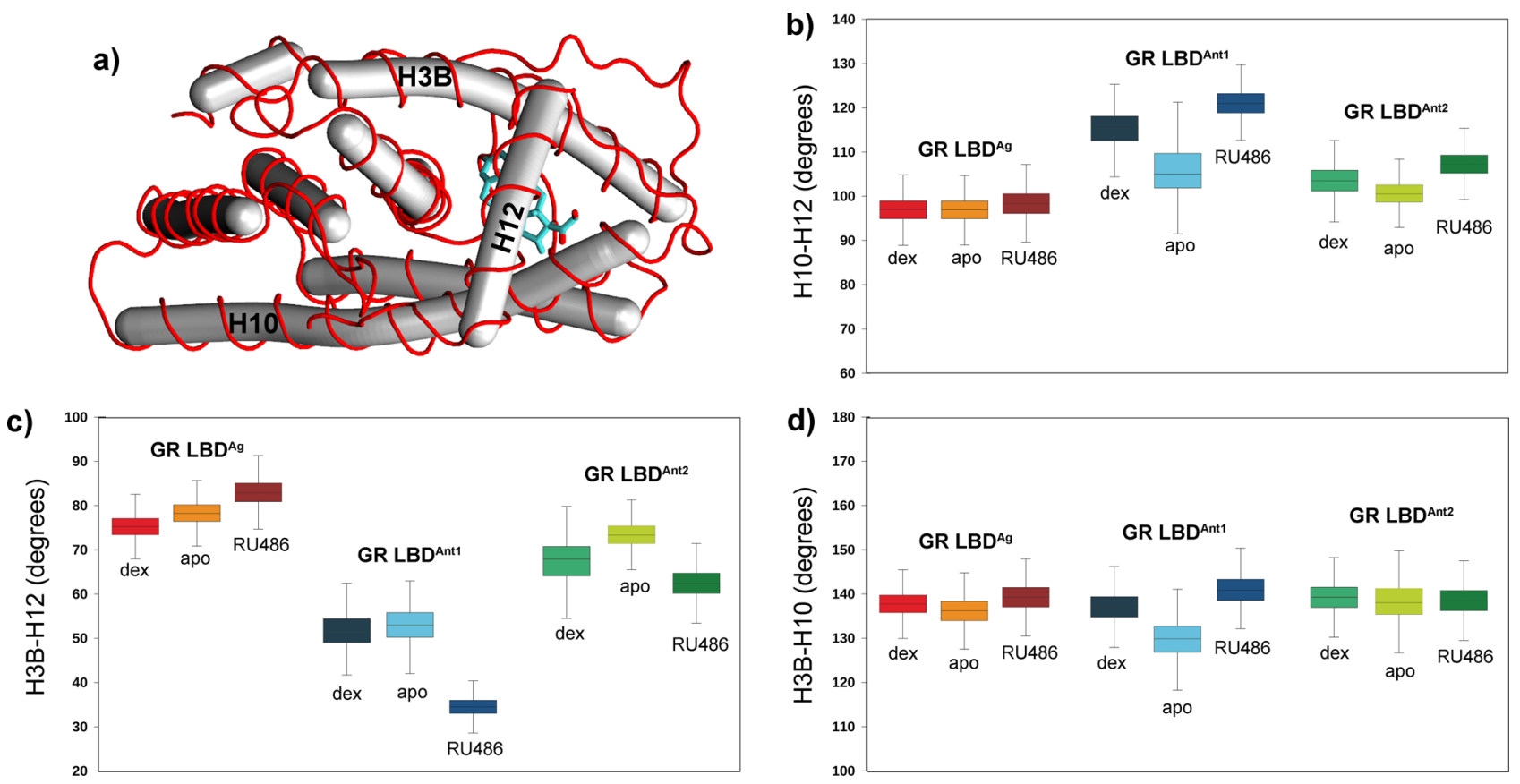

Figure 5. a) Overall structure of the GR $\mathrm{LBD}^{\mathrm{Ag}} / \mathrm{dex}$ complex. Gray tubes display helix-forming residues modeled by Bendix. Segments $\mathrm{H} 3 \mathrm{~B}$ and $\mathrm{H} 10$ and the $\mathrm{H} 12$ helix are marked with black letters. Ligand appears in cyan. b-d) Boxplots of the angles formed between H10-H12 (b), H3B$\mathrm{H} 12$ (c), and H3B-H10 (d).

up to $1000 \mathrm{~ns}$ and compared the resulting angle distribution with GR LBD ${ }^{\mathrm{Ag}} / \mathrm{dex}$ and $\mathrm{LBD}^{\mathrm{Ag}} / \mathrm{RU} 486$ 300-500 ns (Figure S4). It was then confirmed that the H12 sustained its drift in the mentioned direction, partially occupying the AF-2 groove. This emerging state was termed pseudo-GR $\mathrm{LBD}^{\mathrm{Ant3}}$ and incorporated within our pool of systems.

For the GR LBD ${ }^{\text {Ant1 }} /$ RU486 complex, histograms showed a tight angle distribution at $30^{\circ}$ for $\mathrm{H} 3 \mathrm{~B}-\mathrm{H} 12$ and $122^{\circ}$ for H10-H12 angles (Figure 6a). By contrast, for the apo and dex systems, the angle distribution approaches that of GR $\mathrm{LBD}^{\mathrm{Ant}} / \mathrm{RU} 486$. Thus, this $\mathrm{H} 12$ conformation presumably describes an intermediate state between the antagonist state GR LBD ${ }^{\mathrm{Ant}} / \mathrm{RU} 486$ and the agonist state GR $\mathrm{LBD}^{\mathrm{Ag}} / \mathrm{dex}$. In this sense, GR $\mathrm{LBD}^{\mathrm{Ant} 2} /$ apo and GR $\mathrm{LBD}^{\mathrm{Ant} 2} /$ dex histograms were remarkably similar to that of $\mathrm{GR} \mathrm{LBD}^{\mathrm{Ag}} / \mathrm{dex}$ and manifested how the intermediate state evolves toward the agonist state when RU486 is removed or replaced by dex.

A schematic diagram depicting the evolution of each simulated system is shown in Figure $6 \mathrm{~b}$. The complexes directly derived from the crystallized structures conserve the $\mathrm{H} 12$ in a position similar to the original one, while variations in LBP condition modify the H12 destination during the MD. Figure 7 shows representative snapshots of the main configurations observed in the $\mathrm{H} 10-\mathrm{H} 12$ and $\mathrm{H} 3 \mathrm{~B}-\mathrm{H} 12$ angle configurational space. It can be observed that while the C-terminal half of the $\mathrm{H} 12$ occupies a similar position in all the systems, its $\mathrm{N}$-terminal half explores different states swinging from virtually blocking the AF-2 domain to releasing and enlarging it.

2.3.3. Dimension of the AF-2 Domain. To estimate how $\mathrm{H} 12$ conformational changes influence the AF- 2 configuration, we calculated the average distance among $\mathrm{C} \alpha$ atoms residues Glu755 (H12), Lys579 (H3), and Asp590 (H4) (Table 2 and Figure 8). These residues, localized within each of the AF-2 domain conforming helices, participate in anchoring the coregulator to the receptor through polar interactions ("charge clamps"). Hence, the relative position between these three residues determines the accessibility through the opening dimension of the AF-2. As expected, the Lys579-Asp590 distance remained equal in all simulated systems, indicating the permanence in the relative disposition of this coregulator's anchor point. In contrast, large variations characterized the distances involving the $\mathrm{H} 12$ residue. Differences of up to 4.4 and $7.5 \AA$ were calculated for the Lys579-Glu755 and Asp590Glu755 pairs, respectively, with GR $\mathrm{LBD}^{\mathrm{Ag}} / \mathrm{RU} 486$ and GR $\mathrm{LBD}^{\text {Ant1 }} / \mathrm{RU} 486$ at the extremes. These findings evidence that the AF-2 dimensions and interactions ultimately depend on H12 spatial configuration.

2.3.4. Ligand Binding Mode. A detailed analysis of the ligand binding mode was pursued calculating hydrogen bond interactions between the polar moieties of dex or RU486 and the polar residues of the GR LBP. Figure S5 displays the hydrogen-bond occupancy (HBO) between ligand and receptor atoms for the last $300 \mathrm{~ns}$ of the trajectories. Globally, in the 6 holo-systems these ligand-receptor interactions occur in well characterized zones, i.e., the C-3 carbonyl group and the 17-hydroxyl group; in addition, dex presents long-lasting hydrogen bonds through its 11- and 21-hydroxyl groups.

The C-3 carbonyl group of both ligands participates in a hydrogen bonding network that involves Gln570 (H3) and Arg611 (H5). In the case of dex, while for the GR LBD ${ }^{\mathrm{Ag}} / \mathrm{dex}$ and GR $\mathrm{LBD}^{\text {Ant1 }} /$ dex complexes the interaction is mainly mediated by a water molecule (explaining in part the small $\mathrm{HBO}$ values for the Arg611), larger direct ligand-receptor contacts are observed for the GR $\mathrm{LBD}^{\mathrm{Ant} 2} / \mathrm{dex}$ complex. For RU486 systems, the agonist state presents similar values for those interactions, opposite to the nonagonist states which show little interaction with Gln570 but a more sustained one with Arg611.

Regarding the 17-hydroxyl group, a persistent hydrogen bond is formed with Gln642 (H7) in the GR LBD ${ }^{\text {Ant1 }} /$ dex and, to some extension, the GR $\mathrm{LBD}^{\mathrm{Ag}} / \mathrm{RU} 486$ systems. It is 

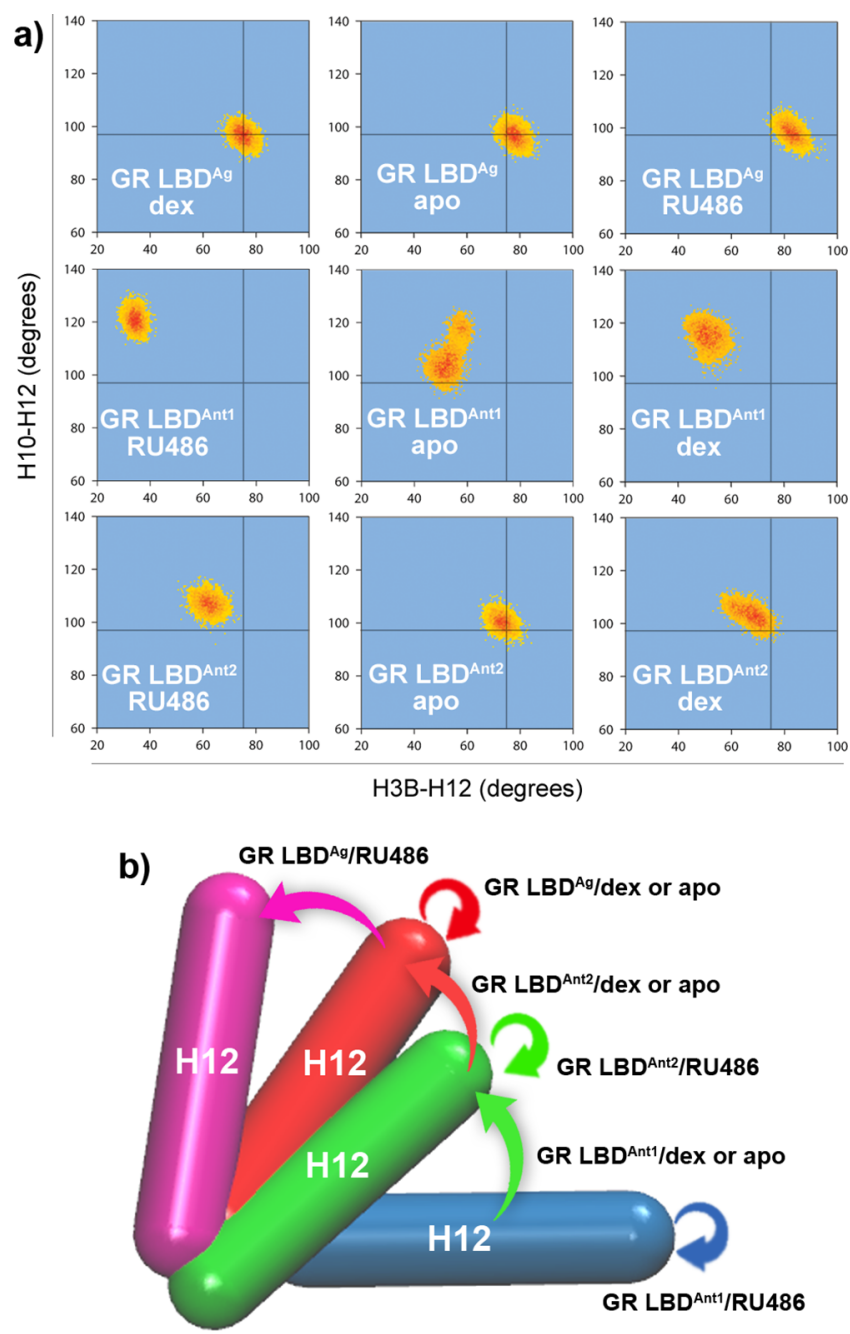

Figure 6. a) Distribution of the $\mathrm{H} 3 \mathrm{~B}-\mathrm{H} 12$ and $\mathrm{H} 10-\mathrm{H} 12$ angles population for the simulated systems. Colors indicate relative number of frames: higher prevalence in red, lower prevalence in yellow. Black crosses reference the GR $\mathrm{LBD}^{\mathrm{Ag}} / \mathrm{dex}$ complex position. b) Schematic diagram of the $\mathrm{H} 12$ evolution along the simulated MD trajectories.

noticeable that the crystal-derived structures only maintain sporadic interaction with this residue, suggesting that those contacts within the mentioned complexes are forced due to the size-occupancy relationship of the LBP and each ligand: a smaller agonist LBP would shift RU486 orientation toward Gln642, while a larger LBP conformation would prevent dex from interacting through its 11- and 21-hydroxyl groups (see below), thus favoring this bond.

For dex 11- and 21-hydroxyl groups, large $\mathrm{HBO}$ values are detected in the agonist GR $\mathrm{LBD}^{\mathrm{Ag}}$ state, particularly between the couples 11-hydroxyl/Asn564 (H3) and 21-hydroxyl/ Thr739 (H11). Although less frequently, these are also present in the GR $\mathrm{LBD}^{\mathrm{Ant} 2} / \mathrm{dex}$ complex; on the contrary, as mentioned before, no interactions between these polar groups and LBP residues are formed in the antagonist GR LBD ${ }^{\text {Ant1 }}$ state. These findings suggest that variations in the $\mathrm{H} 12$ position are related with variations in the mode in which 11and 21-hydroxyl are recognized by the LBP, plus strengthening the notion that the GR $L_{B D}{ }^{A n t 2}$ resembles an intermediate state between GR LBD ${ }^{\mathrm{Ag}}$ and GR LBD ${ }^{\mathrm{Ant} 1}$.

On the other hand, we identified the contact residues for the RU486 dimethylaniline moiety along each GR LBD/RU486

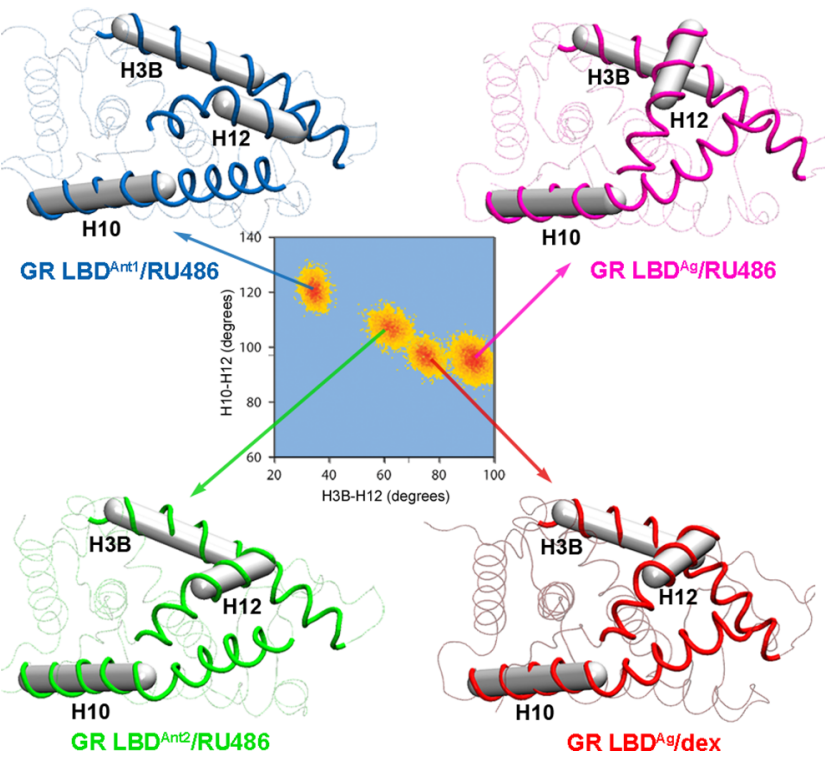

Figure 7. Superposition of the $\mathrm{H} 10-\mathrm{H} 12 / \mathrm{H} 3 \mathrm{~B}-\mathrm{H} 12$ angle's histograms of GR $\mathrm{LBD}^{\mathrm{Ag}} / \mathrm{dex}$, GR LBD $\mathrm{Ag} / \mathrm{RU} 486$ (calculated from the 500-1000 ns interval), GR LBD Ant1/RU486, and GR LBD ${ }^{\text {Ant2 } / ~}$ RU486 complexes and their corresponding representative snapshots depicting the different positions adopted by the H12.

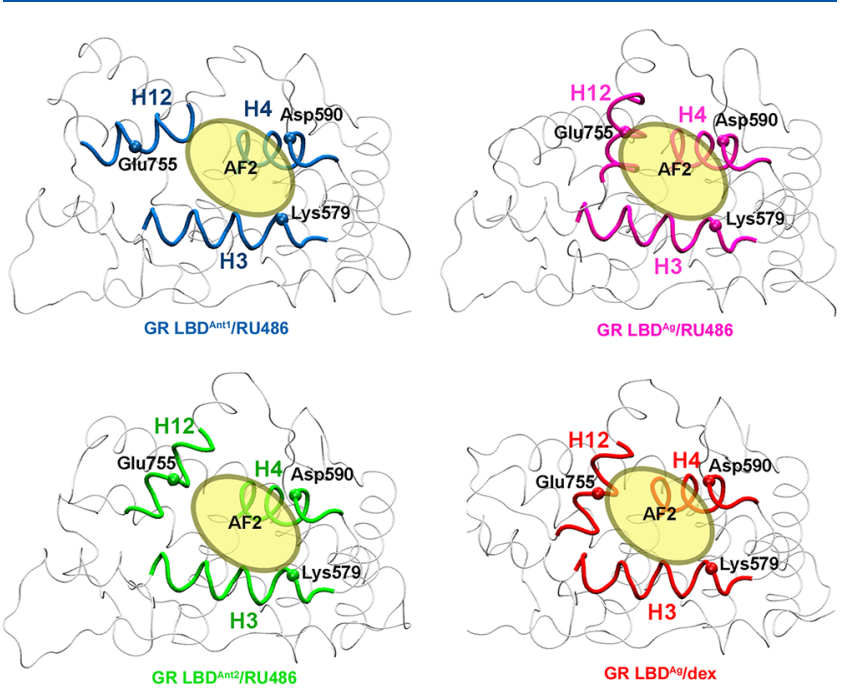

Figure 8. AF-2 conformation in the stated systems. $\mathrm{C} \alpha$ atoms of the receptor residues involved in the "charge clamp" interactions (Lys579, Asp590, and Glu755) are shown as spheres. The position of the AF-2 groove is indicated with a yellow ellipse.

simulation (Figure S6). In the three systems, this voluminous group contacts with Gly567 and Gly568 of the H3 and Trp600 localized between the $\mathrm{H} 4$ and $\mathrm{H} 5$. The third contact point, however, differs for each complex: Phe749 for GR LBD ${ }^{\mathrm{Ag}}$ / RU486, Leu753 for the GR LBD ${ }^{\text {Ant1 }} /$ RU486, and Ile756 for the GR LBD ${ }^{\text {Ant2 }} /$ RU486. Remarkably, the last two belong to the H12, while the first is located within the H11-H12 loop, correlating the contacts established by $\mathrm{H} 12$ residues with the RU486 dimethylaniline moiety and the conformation adopted by the $\mathrm{H} 12$.

On the whole, it is noteworthy that all systems bond with at least one residue of the $\mathrm{H} 3$ chain (which has been earlier proven to be practically invariant), while other interactions are ultimately related to the GR LBD (and particularly H12) 
configuration. This becomes more evident when ligands are switched. On the other hand, the number of interactions is positively correlated with the stability observed in the MDs. Indeed, systems with larger interactions evidenced less fluctuations than those with few contacts along their trajectories, with $\mathrm{GR} \mathrm{LBD}^{\mathrm{Ag}} / \mathrm{dex}$ representing the most stable complex.

2.4. Steered Molecular Dynamics Simulation. Through cMD simulations we have found that the H12 can adopt numerous positions depending on LBP conditions (i.e., ligand identity and occupancy), with the GR $\mathrm{LBD}^{\mathrm{Ag}} / \mathrm{RU} 486$ (or pseudo-GR $\mathrm{LBD}^{\mathrm{Ant}}$ ) at one extreme of the conformational space and the GR LBD ${ }^{\text {Ant } 1} /$ RU486 at the other. Considering the already mentioned limitations of $\mathrm{cMD}$, we alternatively employed steered MD simulations (SMD) to explore putative transitions between those two states. In SMD, an external force is applied to drive the system along a selected reaction coordinate or "collective variable" (CV) at a constant velocity. ${ }^{26}$ The election of an adequate $\mathrm{CV}$ capable to describe the pathway between two states of the protein is mandatory and usually very challenging.

At first, considering our cMD results, angles $\mathrm{H} 12-\mathrm{H} 3 \mathrm{~B}$ and $\mathrm{H} 12-\mathrm{H} 11 \mathrm{~A}$ were taken as $\mathrm{CV}$ to displace the $\mathrm{H} 12$ in the absence of ligand, but trajectories proceeded with considerable deformation in several regions of the receptor. Then, we tested a combination of distances between $\mathrm{C} \alpha$ atoms of key residues from $\mathrm{H} 3, \mathrm{H} 11$, and $\mathrm{H} 12$, also obtaining inadequate pathways. Other geometrical descriptors, such as combinations of distances and angles of the main variable residues, were equally assessed, with no proper results. Finally, when using the RMSD of $\mathrm{H} 3, \mathrm{H} 11$, and $\mathrm{H} 12 \mathrm{C} \alpha$ residues as $\mathrm{CV}$, we obtained a smooth path between the extreme states without constitutional deformations and with well conserved secondary structure elements. Figure 9a shows the temporal evolution of the $\mathrm{CV}$, and Figure $9 \mathrm{~b}$ shows the superposition of snapshots extracted at regular intervals from the $100 \mathrm{~ns}$ SMD trajectory. As it can be observed, along the transition from the pseudo-GR $\mathrm{LBD}^{\mathrm{Ant} 3}$ to the GR $\mathrm{LBD}^{\text {Ant1 }}$, the $\mathrm{H} 12$ visits multiple intermediary conformations, noteworthily including those corresponding to the GR $\mathrm{LBD}^{\mathrm{Ag}}$ and $\mathrm{GR} \mathrm{LBD}^{\mathrm{Ant} 2}$ original states.

SMD trajectories were also simulated for dex and RU486 complexes, finding a similar behavior to the apo system, with straightforward pathways in which both the GR LBD integrity and secondary structure were conserved. All SMD trajectories were also analyzed by monitoring the temporal evolution of the H3B-H12 and H10-H12 angles (Figure S7), finding only some minor discrepancies among all systems. For example, the $\mathrm{H} 3 \mathrm{~B}-\mathrm{H} 12$ angle values in the first half of the simulation were larger in the RU486 complex than in the apo and dex systems, as was expected due to the bulky substituent of the antiglucocorticoid. On the other hand, the H10-H12 angle showed differences between dex and RU486 when the GR $\mathrm{LBD}^{\mathrm{Ant} 2}$ state was reached. As a control, SMD trajectories were also carried out in the reverse direction (from the GR LBD ${ }^{\text {Ant1 }}$ to the pseudo-GR LBD ${ }^{\text {Ant3 }}$ states) in the three conditions (apo, dex, and RU486), finding a very similar behavior to that described in the direct direction (data not shown).

In summary, the one-dimensional path coordinate RMSD successfully illustrates a complete dynamic picture of the conformational space sampled by the H12 in the GR LBD. Hence, this CV was then employed to obtain an energetic profile of the configurational space.
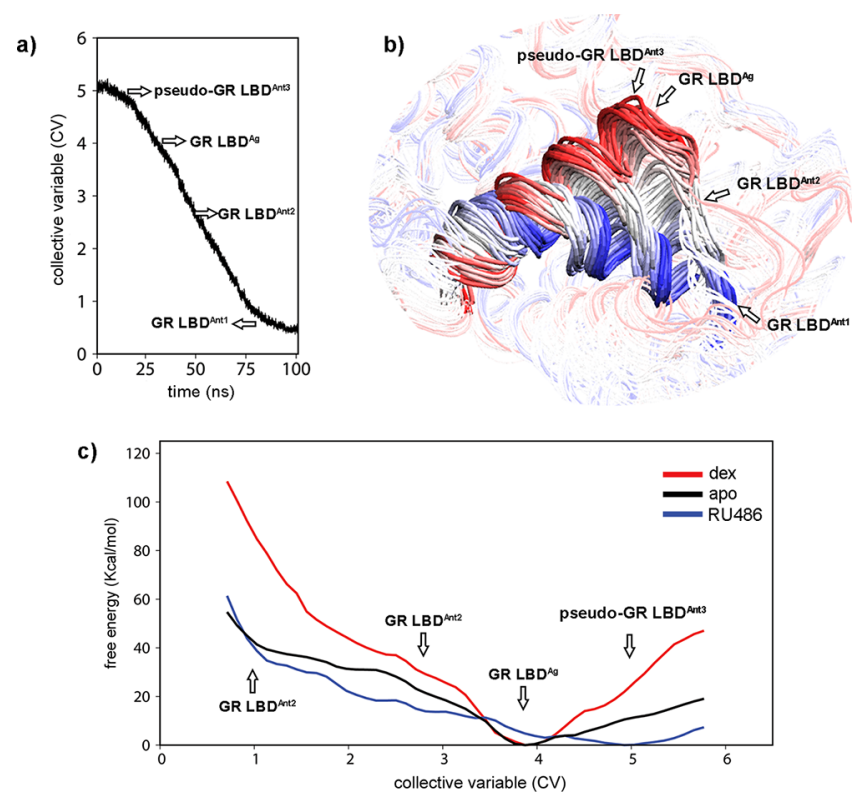

Figure 9. a) Temporal evolution of the collective variable (RMSD of $\mathrm{C} \alpha$ atoms of H3, H11, and H12). b) Superposition of 100 SMD simulation snapshots, sweeping from pseudo-GR $\mathrm{LBD}^{\mathrm{Ant3}}$ to GR $\mathrm{LBD}^{\mathrm{Ant1}}$. c) Free energy surface determined by Umbrella Sampling (GR LBD/dex in red, GR LBD/RU486 in blue, and GR LBD/apo in black). In all graphics, arrows point to the original GR LBD systems' position.

2.5. Umbrella Sampling Analysis. After validating a suitable CV for our systems, we performed an Umbrella Sampling (US) analysis ${ }^{27}$ using the RMSD of H3, H11, and $\mathrm{H} 12 \mathrm{C} \alpha$ atoms as CV. The three SMD trajectories (apo, dex, and RU486) were sampled every $0.2 \AA$ distance along the CV, obtaining 25 snapshots for each system. These were then equilibrated and simulated for 3 ns. The last 2 ns of each window were integrated with the Umbrella Integration method. $^{28}$

The free energy obtained through US was then plotted as a function of the $\mathrm{CV}$, showing a clear difference between GR LBD dex and GR LBD RU486 systems (Figure 9c). Indeed, in the presence of dex, a deep minimum is described at $\mathrm{CV}=3.8$ $\AA$, which corresponds to the $\mathrm{H} 12$ position in the agonistic conformation (GR LBD ${ }^{\mathrm{Ag}}$ original state). Large energetically barriers are observed when the $\mathrm{H} 12$ is displaced toward both GR LBD ${ }^{\text {Ant2 }}$ and pseudo-GR LBD ${ }^{\text {Ant3 }} \mathrm{H} 12$ positions, indicating that this ligand anchors the $\mathrm{H} 12$ in a well-defined and stable position. This finding sustains the large rigidity observed in the cMD simulations and explains the full agonistic activity of this ligand.

In contrast, RU486 provides a flatter free-energy curve, in accordance with the wider flexibility exhibited by the RU486 systems in cMD simulations. A broad global minimum is observed at $\mathrm{CV}=5 \AA$, concordant with the pseudo-GR $\mathrm{LBD}^{\text {Ant3 }} \mathrm{H} 12$ conformation. In turn, GR LBD ${ }^{\text {Ant1 }}$ and GR $\mathrm{LBD}^{\text {Ant2 }} \mathrm{H} 12$ positions do not correlate with any noticeable minima but to plain regions of the free energy surface. The GR $\mathrm{LBD} /$ apo system also provides a flattened free energy curve with a slight global minimun at $\mathrm{CV}=\sim 4 \AA$.

\section{CONCLUSION}

The GR LBD conform to a compact globular structure whose end-terminal helices play a crucial role in the activity of this 
receptor's domain. Thus, following an approach of concatenated MD simulations of different types, we focused on deciphering the correlation between ligand identity and the GR LBD H12 dynamic behavior. Even when only two commercial ligands were evaluated, we consider performing SMD simulations followed by US analysis a powerful strategy and a potential tool to satisfactorily understand the molecular basis of action of glucocorticoids with different activity profiles.

Several GR LBD systems had to be generated starting from the structure of the nonagonist GR LBD/RU486 complex (pdb:3h52). This complex crystallized as a tetramer with the $\mathrm{H} 1$ unfolded in the four monomers (resembling the $\mathrm{H} 1$ situation described for the native GR LBD apo $)^{25}$ and required an in silico refolding in all cases. The straightforward obtention of stable closed structures upon simulation supports the idea that the interaction between monomers during the GR LBD/ RU486 crystallization process prevented the $\mathrm{H} 1$ from adopting its complete final shape. Previous studies assessing the ligand's access/release to the receptor have mostly spotlighted the $\mathrm{H} 6-\mathrm{H} 7$ plasticity as an integral part of the ligand-entry mechanism. ${ }^{29}$ Our findings, in turn, lead us to consider the GR LBD/RU486 unfolded H1, far from being a crystallization artifice, a determinant in ligand association and dissociation from the GR and a very auspicious element in the understanding of this process.

A detailed depiction of how two paradigmatic glucocorticoids affect the $\mathrm{H} 12$ configurational space is also presented. Moreover, a dynamic GR LBD H12 capable of exploring a wide range of clearly identified and characterized conformations is described. Our results reveal pronounced $\mathrm{H} 12$ reorientations depending on the LBP conditions, while the remainder of the GR LBD entails only modest changes in the $\mathrm{N}$-terminal H3 and the C-terminal H11. Together, these findings fit within the conformational-selection model in explaining GR agonism and antagonism.

Potential surface analysis delineated a deep minimum for the GR LBD dex system, indicating that among all possible conformations the agonist dex privileges only a few poses, providing high rigidity to the receptor-ligand complex. As a consequence, the AF-2 domain remains virtually fixed, suitable for recognizing coactivators' LxxLL motifs, as present in NCoAs. Alternatively, in the presence of the antagonist RU486, the H12 conformational amplitude appears to be wider, as indicated by a flatter potential surface energy with no prominent minimum. Antagonist complexes' larger flexibility in comparison to agonist systems has also been described for the androgen $^{23}$ and the estrogen receptors. ${ }^{24}$ Besides, RU486 would also withdraw the $\mathrm{H} 12$ from the agonist state and place it on either side of the ligand's bulky substituent. From these observations, we propose that in the absence of coregulators, the H12 lowest-energy state would be reached when this helix directly occupies the AF-2 groove. In turn, the presence of corepressors would stabilize conformations that extend the AF2 dimensions. Therefore, $\mathrm{H} 12$ conformational selection is a dynamic equilibrium ultimately determined by both the identity of the ligand and the presence of corepressors.

Finally, by characterizing novel $\mathrm{H} 12$ putative conformations different from those already crystallized, we provide a whole series of GR LBD systems that represent potential targets for the rational design of new glucocorticoid drugs, with the ultimate aim of finely modulating the GR activity.

\section{COMPUTATIONAL METHODS}

4.1. Initial GR LBD Structures. Five GR LBD structures were initially built for the MD simulations (Table 1). The GR $\mathrm{LBD}^{\mathrm{Ag}}$ coordinates were taken from the crystal structure of the GR LBD/dex complex (residues 528 to 777 of chain A from pdb:1m2z). GR LBD ${ }^{\text {Ant1 }}$, GR LBD ${ }^{\text {Ant2 }}$, and GR $\operatorname{LBD}^{\text {Ant3 }}$ were constructed by combining coordinates from two crystal structures: residues 528 to 554 of chain A of the GR LBD/ dex complex (pdb:1m2z) and residues 555 to 777 of chain $A$, $\mathrm{B}$, or C of the crystal structure of the GR LBD/RU486 complex (pdb:3h52). The missing regions in GR $\mathrm{LBD}^{\text {Ant2 }}$ and GR LBD $^{\text {Ant3 }}$ (Table 1) were modeled with the Modeller software. ${ }^{30}$ In all cases, the introduced mutations were reverted to retrieve the wild-type GR LBD sequence. Using the GR $\mathrm{LBD}^{\mathrm{Ag}}$, GR LBD ${ }^{\mathrm{Ant} 1}$, and GR LBD ${ }^{\mathrm{Ant} 2}$ coordinates, 9 systems (3 apo and 6 holo) were prepared for classical MD simulations (Table 2). For the GR LBD ${ }^{\mathrm{Ag}} / \mathrm{dex}, \mathrm{GR} \mathrm{LBD}{ }^{\mathrm{Ant}} / \mathrm{RU} 486$, and GR LBD ${ }^{\text {Ant2}} /$ RU486, the crystal coordinates of the ligands were conserved. Ligand's coordinates were directly removed to obtain apo systems, while for the cross-systems GR LBD ${ }^{\mathrm{Ag}}$ /

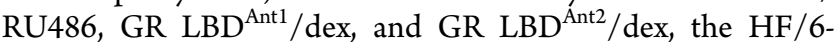
$31 \mathrm{G}^{* *}$ optimized structures of the steroids were introduced into the GR LBD models by superimposing the skeleton carbon atoms of $\mathrm{C}$ and $\mathrm{D}$ rings with the corresponding atoms of the crystallized steroid. For the force field parameters of the ligands, RESP (restraint electrostatic potential) atomic partial charges were computed using the HF/6-31G** method in the quantum chemistry program Gaussian $09^{31}$ on the corresponding optimized structures.

4.2. Classical Molecular Dynamics Simulation. Molecular dynamics were performed with the AMBER 18 software package. ${ }^{32}$ Ligand parameters were assigned according to the general AMBER force field (GAFF) and the corresponding RESP charges using Antechamber. The FF14SB force field parameters were used for receptor residues. Complexes were immersed in an octahedral box of TIP3P water molecules using the Tleap module, giving final systems of about 35,000 atoms. Systems were initially optimized and then gradually heated up to a final temperature of $300 \mathrm{~K}$. All MD production runs of $500 \mathrm{~ns}$ were performed at $300 \mathrm{~K}$ in a NPT ensemble using periodic boundary conditions and the particle mesh Ewald method (grid spacing of $1 \AA$ ) for treating long-range electrostatic interactions with a uniform neutralizing plasma. Temperature regulation was done by using a Langevin thermostat with a collision frequency of $2 \mathrm{ps}-1$. The SHAKE algorithm was used to keep bonds involving $\mathrm{H}$ atoms at their equilibrium length, allowing the use of a 2 fs time step for the integration of Newton's equations.

4.3. Steered Molecular Dynamics Simulation. The RMSD of $\mathrm{C} \alpha$ atoms of $\mathrm{H} 3$ (556 to 579), H10-H11 (711 to 740 ), and $\mathrm{H} 12$ (751 to 766 ) residues was employed as a collective variable $(\mathrm{CV})$ as it is implemented in the Amber package. ${ }^{33}$ The cMD-equilibrated GR $\mathrm{LBD}^{\text {Ant1 }}$ /apo system was used as a reference $(\mathrm{CV}=0 \AA)$ to drive the cMD-equilibrated pseudo-GR $\mathrm{LBD}^{\text {Ant3 }} /$ apo $(\mathrm{CV}=5.1 \AA)$ with a force harmonic constant of $300 \mathrm{kcal} / \mathrm{mol} \AA^{2}$ in a $100 \mathrm{~ns}$ long SMD trajectory. A similar strategy was used to obtain the GR LBD/dex and GR LBD/RU486 SMD trajectories.

4.4. Umbrella Sampling. Twenty-five snapshots with equidistant CV $0.2 \AA$ apart were extracted from the SMD trajectories and prepared for Umbrella Sampling simulations using parameters as for cMD. Production runs of 3 ns were 
performed as for cMD. Only the last 2 ns were integrated through the Umbrella Integration method. ${ }^{28}$

4.5. Analysis of Results. Trajectories were analyzed with CPPTRAJ, ${ }^{34}$ and representative snapshots were visualized with VMD. ${ }^{35}$ The root mean square fluctuations (RMSF) of $\mathrm{C} \alpha$ receptor atoms and boxplots were calculated over the last 300 ns of the cMD trajectories. The angle between two $\alpha$-helices was computed using a vector for each helix. The vector of an $\alpha$ helix was defined by two points determined by the center of mass of four $\mathrm{C} \alpha$ atoms. Residues 568-571 and 576-579 were chosen for the starting and ending points of the $\mathrm{H} 3 \mathrm{~B}$ vector, residues $712-715$ and $718-721$ were chosen for the starting and ending points of the $\mathrm{H} 10$ vector, and residues 751-754 and 761-764 were chosen for the starting and ending points of the $\mathrm{H} 12$ vector. Graphical representations of $\alpha$-helices were obtained with Bendix. ${ }^{36}$ Ramachandran plots were obtained with the RAMPAGE server. ${ }^{37}$ Hydrogen bond occupancy (HBO) for each interaction was calculated with the hbond command of CPPTRAJ using the default parameters for the distance cutoff (acceptor to donor heavy atom less than $3.0 \AA$ ) and for the angle cutoff $\left(135^{\circ}\right)$.

\section{ASSOCIATED CONTENT}

\section{S Supporting Information}

The Supporting Information is available free of charge on the ACS Publications website at DOI: 10.1021/acs.jcim.9b00776.

Figure S1: Ramachandran plots of equilibrated GR $\mathrm{LBD}^{\text {Ant1 }} / \mathrm{RU} 486$ and GR LBD ${ }^{\text {Ant2 } / R U 486 ~ c o m p l e x e s . ~}$ Figure S2: Root mean square fluctuation (RMSF) measured over last $300 \mathrm{~ns}$ of cMD simulations. Figure S3: Time evolution of angle conformed between H3B$\mathrm{H} 12, \mathrm{H} 10-\mathrm{H} 12$, and $\mathrm{H} 3 \mathrm{~B}-\mathrm{H} 10$ helices. Figure S4: Distribution of H3B-H12 and H10-H12 angles. Figure S5: Comparison of dex and RU486 ligand binding mode: schematic representation of polar interactions between ligands and LBP of GR LBD. Figure S6: Residues contacting dimethylaniline moiety of RU486 in GR LBD/RU486 systems. Figure S7: Time evolution of $\mathrm{H} 3 \mathrm{~B}-\mathrm{H} 12$ and H10-H12 angles for GR LBD/dex, GR LBD/apo, and GR LBD/RU486 systems along SMD simulations (PDF)

\section{AUTHOR INFORMATION}

\section{Corresponding Author}

*E-mail: lalvarez@qo.fcen.uba.ar.

\section{ORCID}

Lautaro D. Alvarez: 0000-0001-8841-2330

\section{Author Contributions}

The manuscript was written through contributions of all authors. All authors have given approval to the final version of the manuscript.

\section{Funding}

The authors are thankful for the financial support from Agencia Nacional de Promoción Científica y Tecnológica, Préstamo BID-PICT2014-0630 and BID-PICT2016-0261.

\section{Notes}

The authors declare no competing financial interest.

\section{REFERENCES}

(1) Timmermans, S.; Souffriau, J.; Libert, C. A General Introduction to Glucocorticoid Biology. Front. Immunol. 2019, 10, 1545.
(2) Weikum, E. R.; Knuesel, M. T.; Ortlund, E. A.; Yamamoto, K. R. Glucocorticoid receptor control of transcription: precision and plasticity via allostery. Nat. Rev. Mol. Cell Biol. 2017, 18, 159-174.

(3) Desmet, S. J.; De Bosscher, K. Glucocorticoid receptors: finding the middle ground. J. Clin. Invest. 2017, 127, 1136-1145.

(4) Escoter-Torres, L.; Caratti, G.; Mechtidou, A.; Tuckermann, J.; Uhlenhaut, N. H.; Vettorazzi, S. Fighting the Fire: Mechanisms of Inflammatory Gene Regulation by the Glucocorticoid Receptor. Front. Immunol. 2019, 10, 1859.

(5) Meijer, O. C.; Koorneef, L. L.; Kroon, J. Glucocorticoid receptor modulators. Ann. Endocrinol. (Paris) 2018, 79 (3), 107-111.

(6) Weikum, E. R.; Liu, X.; Ortlund, E. A. The nuclear receptor superfamily: A structural perspective. Protein Sci. 2018, 27, 18761892.

(7) Veleiro, A. S.; Alvarez, L. D.; Eduardo, S. L.; Burton, G. Structure of the glucocorticoid receptor, a flexible protein that can adapt to different ligands. ChemMedChem 2010, 5 (5), 649-659.

(8) Kojetin, D. J.; Burris, T. P. Small molecule modulation of nuclear receptor conformational dynamics: implications for function and drug discovery. Mol. Pharmacol. 2013, 83 (1), 1-8.

(9) Rastinejad, F.; Huang, P.; Chandra, V.; Khorasanizadeh, S. Understanding nuclear receptor form and function using structural biology. J. Mol. Endocrinol. 2013, 51 (3), T1-T21.

(10) Gampe, J.; Montana, V. G.; Lambert, M. H.; Wisely, G. B.; Milburn, M. V.; Xu, H. E. Structural basis for autorepression of retinoid X receptor by tetramer formation and the AF-2 helix. Genes Dev. 2000, 14 (17), 2229-2241.

(11) Batista, M. R. B.; Martínez, L. Dynamics of nuclear receptor Helix-12 switch of transcription activation by modeling time-resolved fluorescence anisotropy decays. Biophys. J. 2013, 105 (7), 1670-1680.

(12) Michel, D. Conformational selection or induced fit? New insights from old principles. Biochimie 2016, 128-129, 48-54.

(13) Chrisman, I. M.; Nemetchek, M. D.; De Vera, I. M. S.; Shang, J.; Heidari, Z.; Long, Y.; Reyes-Caballero, H.; Galindo-Murillo, R.; Cheatham, T. E.; Blayo, A. L.; Shin, Y.; Fuhrmann, J.; Griffin, P.; Kamenecka, T. M.; Kojetin, J.; Hughes, T. S. Defining a conformational ensemble that directs activation of PPAR $\gamma$. Nat. Commun. 2018, 9 (1), 1794.

(14) Bledsoe, R. K.; Montana, V. G.; Stanley, T. B.; Delves, C. J.; Apolito, C. J.; McKee, D. D.; Consler, T. G.; Parks, D. J.; Stewart, E. L.; Willson, T. M.; Lambert, M. H.; Moore, J. T.; Pearce, K. H.; Xu, H. E. Crystal structure of the glucocorticoid receptor ligand binding domain reveals a novel mode of receptor dimerization and coactivator recognition. Cell 2002, 110 (1), 93-105.

(15) Kauppi, B.; Jakob, C.; Färnegårdh, M.; Yang, J.; Ahola, H.; Alarcon, M.; Calles, K.; Engström, O.; Harlan, J.; Muchmore, S.; Ramqvist, A. K.; Thorell, S.; Ohman, L.; Greer, J.; Gustafsson, J. A.; Carlstedt-Duke, J.; Carlquist, M. The three-dimensional structures of antagonistic and agonistic forms of the glucocorticoid receptor ligandbinding domain: RU-486 induces a transconformation that leads to active antagonism. J. Biol. Chem. 2003, 278 (25), 22748-22754.

(16) Schoch, G. A.; D’Arcy, B.; Stihle, M.; Burger, D.; Bär, D.; Benz, J.; Thoma, R.; Ruf, A. Molecular switch in the glucocorticoid receptor: active and passive antagonist conformations. J. Mol. Biol. 2010, 395 (3), 568-577.

(17) Maximova, T.; Moffatt, R.; Ma, B.; Nussinov, R.; Shehu, A. Principles and Overview of Sampling Methods for Modeling Macromolecular Structure and Dynamics. PLoS Comput. Biol. 2016, 12 (4), No. e1004619.

(18) Hollingsworth, S. A.; Dror, R. O. Molecular Dynamics Simulation for All. Neuron 2018, 99 (6), 1129-1143.

(19) Bernardi, R. C.; Melo, M. C. R.; Schulten, K. Enhanced sampling techniques in molecular dynamics simulations of biological systems. Biochim. Biophys. Acta, Gen. Subj. 2015, 1850 (5), 872-877.

(20) Spyrakis, F.; Barril, X.; Luque, F. J. Molecular Dynamics: a Tool to Understand Nuclear Receptors. In Computational Approaches to Nuclear Receptors; Cozzini, P., Kellogg, G. E., Eds.; Royal Society of Chemistry: 2012; pp 60-83. 
(21) Zheng, L.; Lin, V. C.; Mu, Y. Exploring Flexibility of Progesterone Receptor Ligand Binding Domain Using Molecular Dynamics. PLoS One 2016, 11 (11), No. e0165824.

(22) Jin, Y.; Duan, M.; Wang, X.; Kong, X.; Zhou, W.; Sun, H.; Liu, H.; Li, D.; Yu, H.; Li, Y.; Hou, T. Communication between the Ligand-Binding Pocket and the Activation Function-2 Domain of Androgen Receptor Revealed by Molecular Dynamics Simulations. J. Chem. Inf. Model. 2019, 59 (2), 842-857.

(23) Duan, M.; Liu, N.; Zhou, W.; Li, D.; Yang, M.; Hou, T. Structural Diversity of Ligand-Binding Androgen Receptors Revealed by Microsecond Long Molecular Dynamics Simulations and Enhanced Sampling. J. Chem. Theory Comput. 2016, 12 (9), 46114619.

(24) Ng, H. L. Simulations reveal increased fluctuations in estrogen receptor-alpha conformation upon antagonist binding. J. Mol. Graphics Modell. 2016, 69, 72-77.

(25) Suren, T.; Rutz, D.; Mößmer, P.; Merkel, U.; Buchner, J.; Rief, M. Single-molecule force spectroscopy reveals folding steps associated with hormone binding and activation of the glucocorticoid receptor. Proc. Natl. Acad. Sci. 2018, 115 (46), 11688-11693.

(26) Do, P. C.; Lee, E. H.; Le, L. Steered Molecular Dynamics Simulation in Rational Drug Design. J. Chem. Inf. Model. 2018, 58 (8), $1473-1482$.

(27) Kästner, J. Umbrella Sampling. WIREs Comput. Mol. Sci. 2011, $1,932-942$.

(28) Kästner, J.; Thiel, W. Bridging the gap between thermodynamic integration and umbrella sampling provides a novel analysis method: "Umbrella integration. J. Chem. Phys. 2005, 123 (14), 144104.

(29) Edman, K.; Hosseini, A.; Bjursell, M. K.; Aagaard, A.; Wissler, L.; Gunnarsson, A.; Kaminski, T.; Köhler, C.; Bäckström, S.; Jensen, T. J.; Cavallin, A.; Karlsson, U.; Nilsson, E.; Lecina, D.; Takahashi, R.; Grebner, C.; Geschwindner, S.; Lepistö, M.; Hogner, A. C.; Guallar, V. Ligand Binding Mechanism in Steroid Receptors: From Conserved Plasticity to Differential Evolutionary Constraints. Structure 2015, 23 (12), 2280-2290

(30) Webb, B.; Sali, A. Comparative Protein Structure Modeling Using MODELLER. Curr. Protoc. Bioinformatics 2016, 54, 5.6.15.6.37.

(31) Frisch, M. J.; Trucks, G. W.; Schlegel, H. B.; Scuseria, G. E.; Robb, M. A.; Cheeseman, J. R.; Scalmani, G.; Barone, V.; Petersson, G. A.; Nakatsuji, H.; Li, X.; Caricato, M.; Marenich, A.; Bloino, J.; Janesko, B. G.; Gomperts, R.; Mennucci, B.; Hratchian, H. P.; Ortiz, J. V.; Izmaylov, A. F.; Sonnenberg, J. L.; Williams-Young, D.; Ding, F.; Lipparini, F.; Egidi, F.; Goings, J.; Peng, B.; Petrone, A.; Henderson, T.; Ranasinghe, D.; Zakrzewski, V. G.; Gao, J.; Rega, N.; Zheng, G.; Liang, W.; Hada, M.; Ehara, M.; Toyota, K.; Fukuda, R.; Hasegawa, J.; Ishida, M.; Nakajima, T.; Honda, Y.; Kitao, O.; Nakai, H.; Vreven, T.; Throssell, K.; Montgomery, J. A., Jr.; Peralta, J. E.; Ogliaro, F.; Bearpark, M.; Heyd, J. J.; Brothers, E.; Kudin, K. N.; Staroverov, V. N.; Keith, T.; Kobayashi, R.; Normand, J.; Raghavachari, K.; Rendell, A.; Burant, J. C.; Iyengar, S. S.; Tomasi, J.; Cossi, M.; Millam, J. M.; Klene, M.; Adamo, C.; Cammi, R.; Ochterski, J. W.; Martin, R. L.; Morokuma, K.; Farkas, O.; Foresman, J. B.; Fox, D. J. Gaussian 09, Revision A.02; Gaussian, Inc.: Wallingford, CT, 2016.

(32) Case, D. A.; Ben-Shalom, I. Y.; Brozell, S. R.; Cerutti, D. S.; Cheatham, T. E., III; Cruzeiro, V. W. D.; Darden, T. A.; Duke, R. E.; Ghoreishi, D.; Gilson, M. K.; Gohlke, H.; Goetz, A. W.; Greene, D.; Harris, R.; Homeyer, N.; Izadi, S.; Kovalenko, A.; Kurtzman, T.; Lee, T. S.; LeGrand, S.; Li, P.; Lin, C.; Liu, J.; Luchko, T.; Luo, R.; Mermelstein, D. J.; Merz, K. M.; Miao, Y.; Monard, G.; Nguyen, C.; Nguyen, H.; Omelyan, I.; Onufriev, A.; Pan, F.; Qi, R.; Roe, D. R.; Roitberg, A.; Sagui, C.; Schott-Verdugo, S.; Shen, J.; Simmerling, C. L.; Smith, J.; Salomon-Ferrer, R.; Swails, J.; Walker, R. C.; Wang, J.; Wei, H.; Wolf, R. M.; Wu, X.; Xiao, L.; York, D. M.; Kollman, P. A. AMBER 2018; University of California: San Francisco, 2018.

(33) Coutsias, E. A.; Seok, C.; Dill, K. A. Using quaternions to calculate RMSD. J. Comput. Chem. 2004, 25 (15), 1849-1857.
(34) Roe, D. R.; Cheatham, T. E. PTRAJ and CPPTRAJ: Software for Processing and Analysis of Molecular Dynamics Trajectory Data. J. Chem. Theory Comput. 2013, 9 (7), 3084-3095.

(35) Humphrey, W.; Dalke, A.; Schulten, K. VMD: Visual Molecular Dynamics. J. Mol. Graphics 1996, 14 (1), 33-38.

(36) Dahl, A. C. E.; Chavent, M.; Sansom, M. S. P. Bendix: intuitive helix geometry analysis and abstraction. Bioinformatics 2012, 28 (16), 2193-2194.

(37) Lovell, S. C.; Davis, I. W.; Arendall, W. B.; de Bakker, P. I. W.; Word, J. M.; Prisant, M. G.; Richardson, J. S.; Richardson, D. C. Structure validation by Calpha geometry: phi, psi and Cbeta deviation. Proteins: Struct., Funct., Genet. 2003, 50 (3), 437-450. 